\title{
escapED: A Framework for Creating Educational Escape Rooms and Interactive Games For Higher/Further Education.
}

\author{
Samantha Clarke ${ }^{1}$, Daryl J. Peel ${ }^{2}$, Sylvester Arnab ${ }^{1}$, Luca Morini ${ }^{1}$, Helen Keegan ${ }^{1}$, \\ Oliver Wood ${ }^{1}$ \\ ${ }^{1}$ Disruptive Media Learning Lab, Coventry University, Samantha.clarke@coventry.ac.uk \\ \{Sylvester.arnab, Luca.Morini, Helen.keegan, OliverWood\}@coventry.ac.uk \\ ${ }^{2}$ University of Southampton, D.J.Peel@soton.ac.uk
}

\begin{abstract}
Game-based learning (GBL) is often found to be technologically driven and more often than not, serious games for instance, are conceptualised and designed solely for digital platforms and state of the art technologies. To encourage a greater discussion on the potential benefits and challenges of a more holistic approach to developing $G B L$ that promote human centered interactions and play for learning, the authors present the escapED programme. The escapED programme was conceived following the recent entertainment trend of escape rooms and is used for developing nondigital GBL approaches within education. escapED aids the design and creation of educational Escape Rooms and Interactive Gaming Experiences for staff and students in further/higher education settings. The paper first presents a pilot study that was used to assess the feasibility and acceptance of University teaching staff of embedding interactive $G B L$ into a higher education environment. The authors then present the escapED theoretical framework that was used to create the prototype game for the pilot study as a tool to aid future design and development of on-site interactive experiences. The paper also presents an external developer report of using the escapED framework to develop a prototype game for teaching research methods to Southampton University students. Finally, the authors present a discussion on the use of the escapED framework so far and plans for future work and evaluation in order to provide engaging alternatives for learning and soft skills development amongst higher education staff and students.
\end{abstract}

Keywords: Escape Rooms, Education, Game-based Learning;

\section{Introduction}

The paradigm of adopting games and play as systems for representing and simulating real-life conditions, imparting knowledge and moral teachings, and generally nurturing social evolution, is an approach which has only recently started being referred to as game-based learning (GBL; a denomination originated with Prensky's [1]), but that has in fact existed under very different guises for thousands and thousands of years.

Historical evidence of early (board) games can in fact be dated back to the Predynastic burial tombs in Egypt, c. 3500 BC, where examinations of ancient burial frescos have revealed the oldest game currently known to man: Senet [2], a game meant to symbolize (and possibly teach) the thencurrent theological perspective on the transition to the afterlife. The identification of Senet and other early examples of board games found in various cultures and societies throughout history and wildly different contexts (e.g. The Royal Game of Ur in Mesopotamia, Go in China and Patolli in precolumbian Mesoamerican cultures) emphasizes the fascination and value that humankind has had, and continues to have regarding the nature of games and play, so much that they are deeply embedded into our cultural history as a civilization [3]. The classic discussion of play and culture brought forward from Huizinga in his seminal "Homo Ludens" [4] even reverses the intuitive 
chronology of history and games, in that, he argues, it is in fact culture and civilization that themselves arise in and from "the play spirit".

Even before civilization itself, the close relationship between play and learning can be easily highlighted throughout the animal kingdom. In particular, social play [5], is a behavior present in most extant mammals, and that scaffolds the development of practical skills, behaviors and experiences needed for later adult life, while keeping them flexible and beyond mere instinctual reflex. In discussing further animal play across a number of species, anthropologist Gregory Bateson [6] even argues that the whole system of sociality and meaningful communication that we can now observe in animals did arise and evolve from play.

Whilst it is therefore evident that traditional games, and more in general play, have always existed and have a key role within the context and evolution of life and society on Earth, the 20th century led the way into something new that would deeply change the ways we engage with games and play forever: the rise of the digital revolution. The onset of the digital revolution brought with it computer based technologies that quickly became openly available to a large proportion of humanity (even though issues of digital divide and inequality persist to the present day); video games, simulations and the internet soon followed these technologies, see Parikka, [7] for a critical history of digital media, and offered exciting and novel ways of connecting and engaging with people of all ages, genders, cultures and races across the globe [8].

Still, even further down the line into the 21 st century, humankind's obsession with technology has not waned, and has in fact developed into new ways to experience the world through the use of technology such as; Location-Based Experiences, Smart Phone Technologies, VR, 3D, Augmented Reality and 360 video to name a few, has continued to grow and become central features or provide functional assistance within everyday living. Perhaps one of the most note-worthy aspects of humanity's pursuit of technological ascendancy however, is how the "play spirit" discussed by Huizinga [4] adapts, and the consequent endeavor to adopt these technologies for the purposes of games and play.

The appeal and engagement of video games has long been documented (see, among others, Vorderer \& Bryant, [9] for an in-depth study of digital games' appeal), and statistics produced by Newzoo and GlobalCollect [10] confirmed their far-reaching popularity, as it has been estimated that there are at least 1.78 billion gamers worldwide as of August 2014, with a global market expected to break the hundred billion thresholds of total worth by the end of 2017. Due to the high uptake of computer games throughout society, particularly amongst children and young adults and with the audience quickly broadening to include formerly sidelined segments of the population, such as adult women and senior citizens; see Nichols [11], researchers and educators have championed the potential reach and use of computer games, simulations and their related technologies, both as instructional delivery systems for various learning and behavior change endeavors [12] and as playground through which to let learners freely explore subjects and themes $[13,14]$

As such GBL began to take on a whole new identity which, maybe unreflexively, started to highlight and strongly lean toward digital technology as a central theme [15-17], thereby sidelining in the educational debate other forms of play and games. This trend started in 2001, when Prensky [18] popularised the field as Digital Game-Based Learning, which in turn deeply influenced how the academic world worked upon, developed and perceived the required conditions of GBL, particularly in relation to the requirement of technology. As such, the premise of GBL has since subsequently developed predominantly within academic practice to mean the theory and practices of adopting digital games and technology as a method for delivering learning materials through play and study of in-game components [14, 19, 20]. Tang et al., [21] offer the following definition of GBL that is eminently influenced by this specific, and exclusively digitally oriented perspective, and paradigmatic of the mainstream of this field of research:

"Games-based learning takes advantage of gaming technologies to create a fun, motivating, and interactive virtual learning environment that promotes situated experiential learning. " [21]

As such, most of the current research that has been conducted concerning the practice of GBL centers on the adoption of various technologies and digital gaming preferences as a means to delivering educational content and exploring techniques of maintaining motivation and engagement of varying user types, often at the detriment of different materials, approaches and pedagogies. While indeed it is assumed that an overwhelming proportion of GBL research has been aimed at digital gaming and technology, it has however also been recognized within the academic community that GBL can mean the adoption of non-digital examples such as tabletop, card, board or role playing 
games [22-24]. Despite these emerging examples, research into the design, development and evaluation of non-digital GBL applications still remains much scarcer than research surrounding their digital counterparts, further compounding issues of pedagogical narrowness and digital divide (see Linderoth, [25], for a discussion of the broader ecologies of play excluded by mainstream GBL research).

To hereby challenge the current, still exceedingly dominant, inclination towards adopting digital games and technologies as the leading method of delivering and exploring GBL, the authors present and review the holistic methods toward inclusive, learning oriented game design and development as offered by the GameChangers Programme (http://gamify.org.uk), an Open Game Design community and resource hub built within Coventry University's Disruptive Media Learning Lab (http://dmll.org.uk). The GameChangers Programme and one of its sub-projects titled escapED are hereby discussed and theorized as paradigmatic of how these examples and broad, inclusive approaches could be adopted and built upon to promote a return to inclusive, human centered interaction and play within GBL, rather than the trending technology focused approach that has become a mostly un-reflected upon mainstream as of late.

\section{The escapED Programme}

The escapED programme is a smaller project under the Game Changers initiative (gamify.org.uk). The Game Changers initiative was set up by the Disruptive Media Learning Lab, Coventry University, to explore, experiment and exploit game design thinking in fostering creative problem solving and cross-disciplinary design collaboration. The overarching pedagogical construct of the initiative is motivated by 'learning by designing', which is a project-based inquiry approach, exploiting the characteristics of a design process that is non-linear, iterative, generative and creative. Design thinking as a domain has crossed over to learning, where it is a "way of finding human needs and creating new solutions using the tools and mindsets of design practitioners" [26].

The philosophy and purpose of the escapED programme, is to conceptualize interactive experiences and aid other educational facilitators in creating their own, live-action games for the purposes of education and positive behavior change in higher/further education settings. Based upon the design and experience of traditional entertainment Escape Rooms, escapED is adapted to incorporate educational themes, tangible learning objectives and behavior change metrics, to offer a new approach to human centered GBL. A study conducted by Nicholson [27] on the growing phenomena of Escape Rooms defined them as:

"live-action team-based games where players discover clues, solve puzzles, and accomplish tasks in one or more rooms in order to accomplish a specific goal (usually escaping from the room) in a limited time”. [27]

Traditional entertainment focused Escape Rooms, are growing in popularity worldwide, with rooms now available across most continents including Europe, America and Asia. Nicholson [27] observes that the earliest well- documented example of an Escape Room, was run by the Scrap company in Kyoto, Japan in 2007 which facilitated teams of around 5-6 players. From there on, these live, interactive games have grown to become more elaborate and multi-faceted in their own quest to produce the most engaging and enjoyable experiences to accommodate small teams of a few people, to thousands of players participating in event settings.

Themes and narratives for entertainment Escape Rooms have ranged from horror (zombies, murderers, haunted houses) to mystery and adventure (detective, Indiana Jones). These themes and narratives, set the games atmosphere and lay the foundations of emotional investment and curiosity within the player. Based on live-action role-play (LARP) games such as Dungeons and Dragons (DND) [28] the players essentially take on roles of different characters, although usually the same role such as a hostage, detective etc., within the Game Masters (Escape Room facilitator's) story. A sense of urgency and risk is usually maintained throughout the experience through the methods of time management, actors and props and outside influences, to generate a passionate response and investment from the players into completing the challenge in time.

Data collected from a survey of 175 Escape Rooms from around the world, conducted by Nicholson [27], found that the demographic data of these Escape Rooms players, indicated some noteworthy discussions. Nicholson found that the demographic data from these rooms, exhibited an equal draw of players from both genders. Of the player groups of these rooms, it was reported that $71 \%$ were mixed genders, $15 \%$ were all male and $14 \%$ were all female. If Escape Rooms and other 
interactive, LARP games attract a balanced gender pool of player types, then GBL designers should consider and ask, what are the elements that make these experiences truly appealing to both genders?

Based on the growing market and the documented engagement levels that Escape Rooms seem to have generated within their diverse types of players, the authors selected to test the appeal and educational value of these forms of games within a University setting and developed a prototype experience of escapED for a University staff training event.

\section{The escapED Pilot}

The educational objective of the pilot game was for players to develop soft skills such as communication, leadership and teamwork throughout their experience. The theme of the game was based around disarming a bomb, which was done through releasing an engineer hostage. Players were tasked to solve riddles and tests to find the key code that would release the hostage's locks.

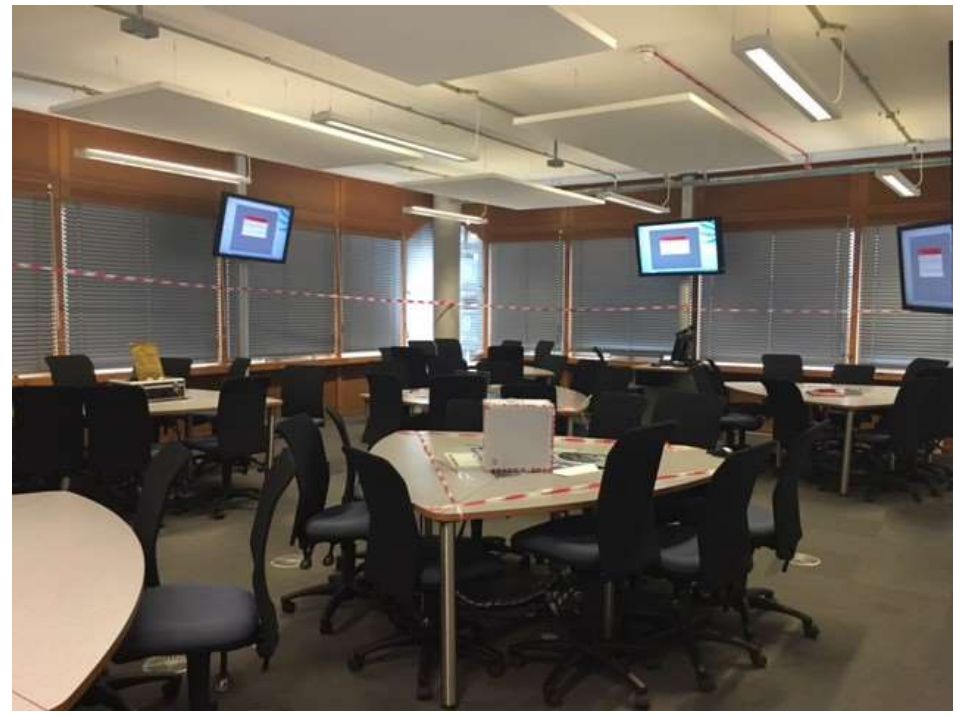

Figure 1 EscapED Prototype: Room 1 (Bomb Room)

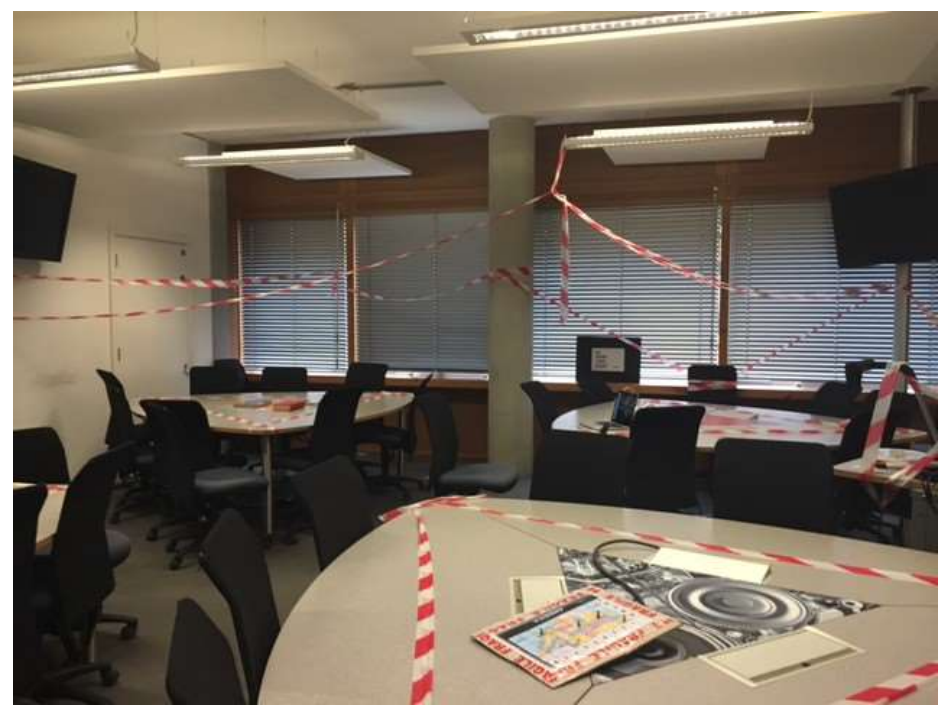

Figure 2 EscapED Prototype: Room 2 (Hostage Room) 
On the day of the event, members of staff signed up to time slots and were put into teams no larger than 6 players. 3 teams participated in the game, with an overall total of 13 players taking part in the event. Each event lasted around 30 minutes, 10 minutes for introduction and rules, and 20 minutes for the game. A key feature of the design of the pilot game was that the teams were split into two groups and placed into two adjoining rooms. One room held the bomb, and the other held the hostage. Riddles and clues were then split between the two rooms and relied on the communication of the players to describe and put the pieces together from both rooms. This requires the team to demonstrate communication skills within such a limited period of time. Players were not allowed to go between rooms and could only communicate via two laptops that were connected to skype, of which one was assigned to each room. Players could not move or touch the laptops, but could bring clues and puzzles to the laptop to show their teammates based in the other room. A first-year drama student was employed to play the part of the hostage and to provide time awareness and clues to the players throughout the game. Most of the players were not aware/had not heard of Escape Room games before the event.

All players were observed by members of the research team at the Disruptive Media Lab via their own connection to skype, and were monitored to observe player engagement and progress within the game and to provide support throughout the game where necessary. Each team was observed to display a similar method of entering and familiarising themselves with the game rooms, displaying conservative behavior but quickly figuring out where the laptops were placed and whether the other participants in the adjoining room could hear them. All players of each of the three teams that participated in the pilot study were observed as displaying high levels of engagement throughout the experience, although this diminished somewhat when the players knew they had less than a minute left to complete the room. One team, was observed to develop a strategy in which they had a designated a main communicator who would be responsible for relaying the information to the other participants in the adjoining room. Here, leadership skill is essential to ensure that informations are collated and communicated effectively between the two teams. Delegation of work was demonstrated as there were various interconnected puzzles and clues in the two rooms. None of the teams that participated in the pilot successfully completed the game experience, however a prize was offered to the team that came closest to completing the challenge.

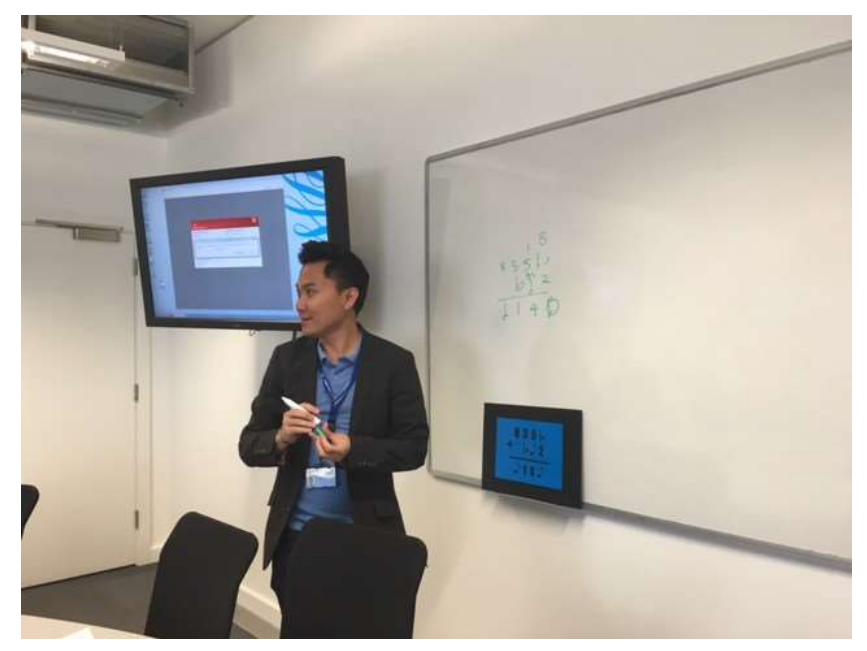

Figure 3 EscapED Prototype: Player interacting with a Puzzle

After the experience, each player was asked to fill in a short feedback sheet that asked four exploratory questions about their experience and perceptions of escapED that are detailed below:

1. Do you think escapED has any educational value?

2. Would you consider using the escapED programme in your lesson plan?

3. What was good about the escapED prototype session?

4. What could we improve?

From the 13 participant players, a total of 8 feedback sheets were returned with all questions answered. All written feedback exhibited a positive theme throughout in regards to the experience 
itself. The words; 'Fun', 'Innovative' and 'Engaging' were repeated throughout the feedback and some players indicated that they did not realize that 20 minutes had passed. All 8 feedback sheets stated that they could see the educational value of escapED, especially if the puzzles and theme of the experience, were worked into their taught subject matter. All feedback sheets indicated that the players would consider using escapED in their lesson plans but were unsure how to facilitate it. A few responses indicated that they thought the experience would be good as an induction into their lessons to encourage getting to know other students. One concern brought up through a number of the feedback responses was that the participants were curious to see how the experience would work with larger groups of players. None of the feedback received suggested that there were improvements that could or should be made to the experience.

Concluding on the pilot trial of escapED, the authors believe that from the behaviours observed within the participating players throughout the live game experience, and from the follow up feedback gathered, that there are significant reasons to explore these types of LARP games for GBL purposes further. It is observed by the authors that from the varied backgrounds of the staff members that took part in the exploratory study of the escapED pilot game, the programme could be used to create and facilitate cross-disciplinary teaching and learning scenarios that are focused on bringing staff and students together from across these disciplines to work and pool their knowledge together to solve and contribute to something greater.

\section{The escapED Framework}

This section presents the theoretical framework for escapED (see Figure 4), which was used to design the prototype game experience for the University staff training event. The framework provides a methodology for creating educational escape rooms and interactive game solutions for learning and behaviour change.

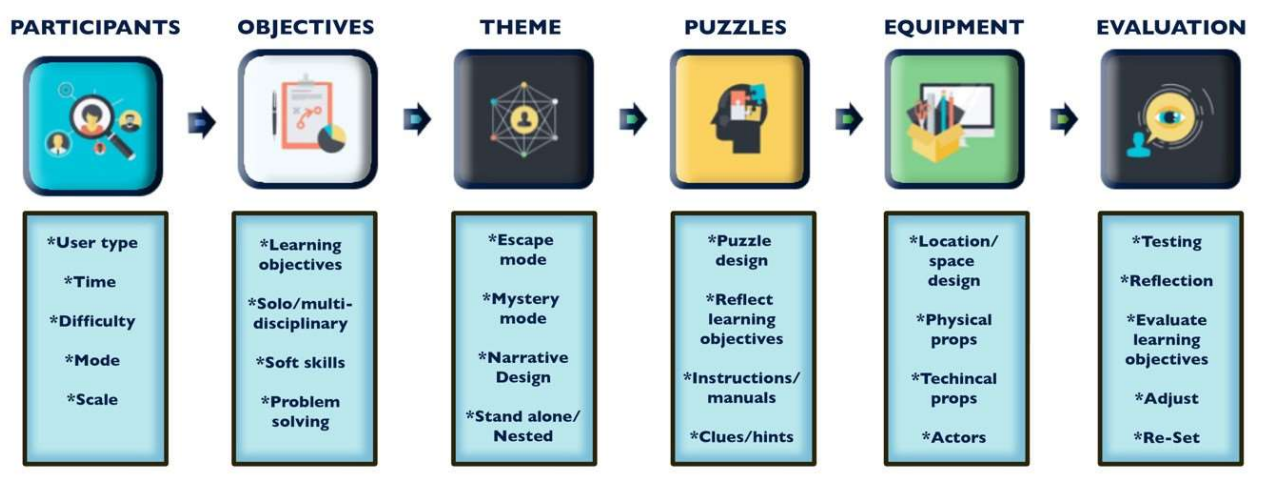

Figure 4 The escapED Framework

Six main areas were considered whilst developing the prototype game for the University training event that consisted of; Participants, Objectives, Theme, Puzzles, Equipment and Evaluation. These areas were influenced and chosen, based on previous works conducted by Arnab \& Clarke, [29], on the considerations of creating trans-disciplinary GBL experiences and work done in a white paper by Nicholson [27]. Each of these areas were further broken down into specific segments that were needed in order to create an educational version of an Escape Room.

\subsection{Participants}

The first step of the escapED framework calls for developers to consider their participants and to in effect, conduct a mini needs analysis of who the game experience is to be targeted towards. Conducting an analysis of the target audience before developing other content is considered conventional practice in most disciplines, and is quite common for entertainment games companies to gather data concerning their player base. Following the stage process suggested from the Trans- 
Disciplinary Methodology by Arnab \& Clarke, [29], the Participants step is broken down into five areas for developers to consider at the start of designing their educational escape room.

- User Type: User needs assessment is carried out to determine player demographic and educational needs.

- Time: Length of experience. Can be short experience of 15 minutes or a longer experience that lasts multiple days.

- Difficulty: Consideration of intended users to scale difficulty of puzzles for different levels of players such as college students, undergrads, post grads, doctorates and staff.

- Mode: Choose mode of experience such as; Cooperation based: Players work together to solve/escape the experience vs Competitive based: Players compete to be the first to figure out the objectives.

- Scale: Choose number of participants the game is to be designed for.

Following this step should provide a base understanding of the user types who will interact with the proposed game and how to proceed with the next development steps.

\subsection{Objectives}

The second step of the escapED framework is to consider developing the learning objectives of the educational escape room experience. Again, following the stage process suggested from the TransDisciplinary Methodology by Arnab \& Clarke, [29], developing the objectives for the game experience early in the design process will ensure that the experience is designed purposefully and that the game theme and puzzles can be developed to enhance the objectives rather than try to embed objectives into an already designed game. The objective step is broken down into four areas for developers to consider.

- Learning Objectives/Behavioural Change Objectives: Learning objectives/behavioural change objectives are required to create a meaningful educational game. These objectives can be worked into the theme, its puzzles and to its chosen mode in order to help structure the learning plan/outcomes. Creating tangible objectives allows to develop the evaluation strategy to assess players learning experience, learning achievements, change metrics and can be iteratively re-designed to focus on the desired outcomes of the experience.

- Solo/Multi-Disciplinary: One discipline or multiple disciplines represented within the game experience.

- Soft Skills: Interactive live-action games can aid development of soft skills such as communication and leadership.

- Problem Solving: Develop problem-solving challenges to make the game experience interesting to players. A range of challenges will appeal to different learner types.

Following this step should provide a foundation in which it is clear what the objectives are that the intended game is trying to achieve with the participants. This will also provide a basis for developing the evaluation strategy later on in the design process.

\subsection{Theme}

The third step of the escapED framework is for developers to consider the overall theme of the experience. In this step, player motivations, game story and content is considered to bring about a compelling game experience for the intended players. Entertainment escape rooms have strong themes and narratives due to the short interaction time that the players have to play through the games (usually an hour). Popular themes such as; detective mysteries, prison breaks, escape the kidnapper, spy/espionage games etc. are used to build believability of the game experience using a range of decorations and props, lighting, music, puzzles and riddles and clues that follow the theme of the room. This step has been informed by the work conducted by Nicholson [27]. The Theme step is split into four areas for developers to consider in their design process.

- Escape Mode: Escape a locked room in a set time.

- Mystery Mode: Solve a mystery in a set time.

- Narrative Design: Develop a compelling narrative for the game to keep player interest.

- Stand-alone/Nested: Determine whether the game is a one-off experience or part of a larger, nested experience in which several games can be designed and played.

Within the four steps, the developers are asked to consider the composition and narrative structure of the game so that players can identify with the game experience and build personal motivations to 
complete the game. It is worth mentioning here, that some players will prefer puzzle experiences with no specific set game theme, however, this is purely a designer choice and preference for how players will experience the game.

\subsection{Puzzles}

The forth step of the escapED framework is for developers to consider developing the puzzles and activities that the players will interact with during the game experience. Puzzles should be designed following the information developed concerning the other steps of the framework particularly the Participants and Objectives steps. This step has been informed by the work conducted by Nicholson [27]).

- Puzzle Design: Puzzles and riddles make a game experience interesting and can be tailored to fit learning objectives.

- Reflect Learning Objectives: Refer to proposed learning objectives and theme to ensure that puzzles reflect the overall goals of the session.

- Instructions/Manuals: Good experiences have clear, set instructions and rules to help guide players.

- Clues/Hints: Escape Rooms are notoriously hard. Insure clues are available and the method of delivering these clues to players in game does not break player immersion.

Ensuring that the puzzles accurately reflect the objectives set previously in the design process will allow for easier validation and assessment of whether the objectives have been achieved at the end of the games experience. It is also useful and an essential part of entertainment escape rooms as noted by Nicholson [27], that the developers provide players with clear instructions and have a plan for providing clues when/if players get stuck whilst playing through the game.

\subsection{Equipment}

The fifth step of the escapED framework is for developers to consider any location/equipment that they are likely to use to support their game experience. These considerations are for practical delivery of the game experience and if informed both by Nicholson's [27] work and the TransDisciplinary Methodology [29]. If the design of the game is to be supported with technology, this step can be useful to consider and plan for how the players are going to interact with that technology and what to do if the technology fails.

- Location/Space Design: Ensure enough space for the game experience and that it is comfortable to move around. The environment should reflect the theme as realistic as possible within means.

- Physical Props: Puzzle props, red-herrings + general environment items, these are needed to make a compelling and workable experience.

- Technical Props: Use technology to enhance the game experience. Computers, VR, Augmented Reality, GPS and location-based identification, can all really bring something to these experiences. However, higher risk of things going wrong/crashing/general not wanting to work/player unfamiliarity.

- Actors: Real-life actors can help concrete the experience further as believable. Actors can also be used as timer indicators or can give out hints if they see the players are getting stuck.

This step is used to bring animation to the game experience in terms of providing a life-like or believable setting for the players to interact with. Following closely with the design considerations from the third step: Theme, developers can quite like a theatre production, 'design their set' and create a rich environment with props, actors or other supporting materials to ensure the experience engages players.

\subsection{Evaluation}

The sixth and final step of the escapED framework is for developers to consider how they are going to evaluate the game experience. Tied closely with the design considerations of the second step: Objectives, the developers are asked to consider the methods that they are going to employ in order to assess whether the game has met the intended objectives and outcomes. This step is informed from the Trans-Disciplinary Methodology by Arnab \& Clarke, [29] in which evaluation is considered to be a main component of developing GBL applications. 
- $\quad$ Testing: Test and iterate the game experience before playing a live session with intended participants.

- Reflection: Reflect with the players of their views and experiences interacting with the game.

- Evaluate Learning Objectives: Create a formal evaluation of the learning objectives that were set for the game experience. This can be done via individual preferred methods.

- Adjust: Use player feedback to provide informed decisions on how adjustments of the game experience.

- Re-set: Create a re-set sheet; a list of all puzzles/riddles and intractable objectives within the game that need to be checked over to ensure they are in the correct state before another play-through.

This step is used to inform the process of gathering data and to assess the overall efficiency and impact of the projects' transfer of knowledge. Additionally, it is useful to use the data gathered from the evaluation to inform any further needed development of the game experience.

The escapED framework presented in this section, is a theoretically informed, first approach at providing a methodology for educational facilitators and other interested parties who wish to create educational escape rooms and live interactive game experiences within higher education settings. Further work by the authors will look at providing a validation study that assesses the quality and efficacy of the escapED framework in terms of usability and usefulness for developers who wish to create their own educational escape rooms.

\section{Using the escapED Framework to Design an Educational Escape Game}

Presented in this section is an account from a Professional Specialist in Learning Design based at the University of Southampton who adopted the escapED Framework in order to create an educational escape game prototype for teaching the subject of research methods to Southampton University students. In this account, he describes the process in which he developed the prototype and how the escapED Framework informed this process.

One issue that is consistently highlighted in higher education, is the need for developed activities that encourage student participation using both elements of extrinsic and intrinsic motivation strategies [30]. This has led to a focus on the use of game-based learning and playful learning design being adopted for higher educational. Colleagues at the University of Southampton, decided to explore the use of Escape Room activities after reading Disruptive Media Learning Lab's "Design your own Educational Escape Room". It was decided to use this framework as a guide for creating a prototype escape game.

The initial step in the framework was to consider 'Participants', this included considering user type, length of play, difficulty and mode. The Escape Room activity that was created would be focused on helping undergraduate first year students reinforce their basic research skills, the box that the game would be created to fit, would also be used for demonstration purposes for academics to understand the development and pedagogy behind the use of educational escape room activities. With this in mind, it was decided to make the activity last a maximum of 30 minutes, this was set using a timer that automatically would begin once the game box was opened. One thing that was difficult to control was the difficulty level of the activity. The box had to be a challenge but not impossible for the participants, meaning that they it was important that they felt they were making a level of progress at all times through the experience. This was achieved by providing various different activities with a range of difficulties in the prototypes design.

The second step of the Framework was to solidify the learning objectives of the activity. As stated before, the prototype activity was developed to foster and develop student research skills. This had to be broken down into more focused learning outcomes. Six learning outcomes were chosen, however, after initial trials of the prototype, it became apparent that six learning outcomes was too many to accommodate in the planned 30 minutes of the prototype experience. The learning outcomes were stripped down, with four main objectives chosen to correspond with four main activities of the game. A variety of activities from paper based information gathering to a physical maze were developed for players to experience in order to achieve the set learning objectives. A variety of activities were utilised so that the experience would stay interesting to the players and would allow for more learning outcomes to be met. 
After identifying the participants needs and solidifying the learning objectives, the Framework suggested to develop the theme. It was advised to make to theme compelling and immersive. This was done by using a theme that is considered consistent with experimental mechanical devices and pseudo-science narratives such as; "Steampunk". To make the theme more engaging and to add depth to the experience, the prototype was also based on the fictional story of "The Island of Dr. Moreau" that surrounds the controversial theme of animal testing, an area in which research methods would fit well thematically.

Originally, it was considered to develop the puzzles at the beginning of the design process, but after following the Disruptive Media Learning Labs framework, it was easier to design the puzzles after solidifying the learning objectives and the overarching theme of the Escape game. This was because it was already known how many puzzles would have to be created, four in all to coincide with the learning objectives. The theme allowed for the use of a specific style of instructions as well as the tone used in clues and hints.

The final step of the framework was the hardest to follow but also the most engaging in terms of designing the overall experience. The location of the prototype was to be a mobile experience, which demanded portable equipment. In order to achieve this, the prototype game was built into a vintage style suitcase as shown in Fig 5 . This meant that the overall experience, theme and realism of the prototype games activities and story could be maintained in any environment and could be transported easily. Finding physical props was done by collecting items from charity shops and government rubbish centers. The prototype did not require any technical props, which made the development process of the activities easier. It was also decided that the whole experience should be able to be run independently from facilitators, which that actors were not used as well.

One final aspect of the guide that was very useful was the idea of briefing and de-briefing participants. The activity was originally run by itself, however, after play testing the activity a few times it was noticed that the participants didn't have any time for reflection on their experience or evaluating the learning objectives which made the activity unsatisfactory. The Escape Room activity now includes both a pre-task and a post-task that facilitates participant interaction and discussion.

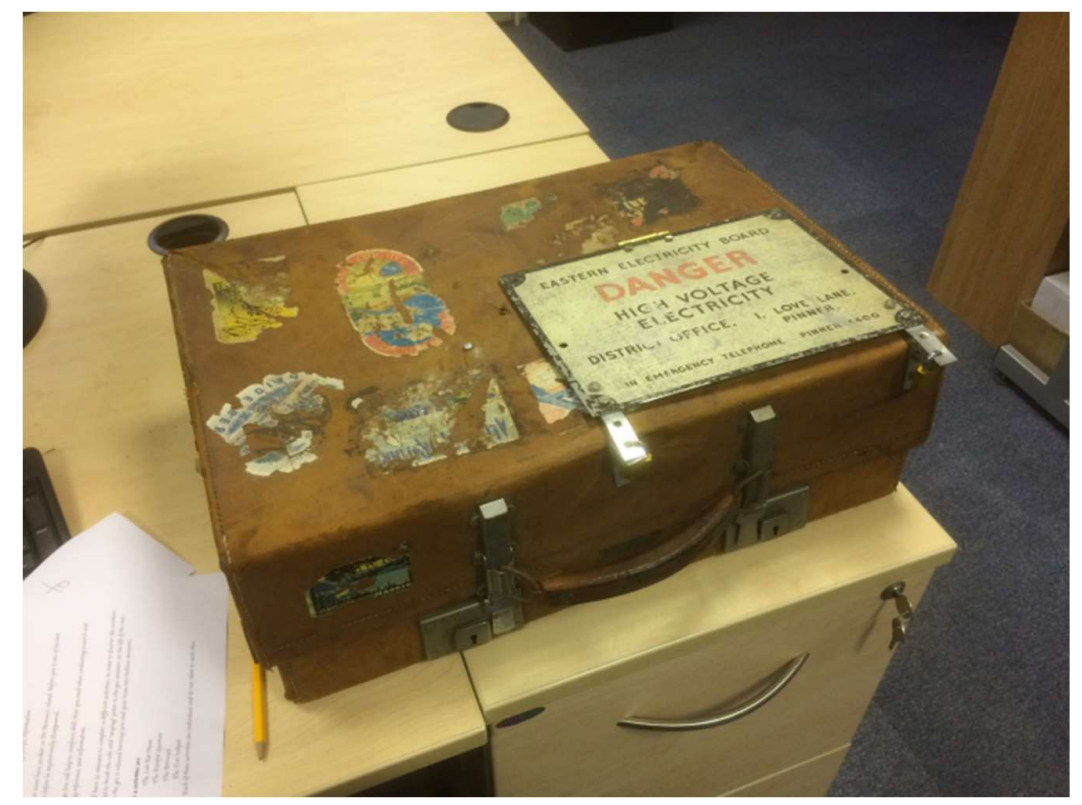

Figure 5 The Island of Dr. Moreau Prototype Game (Outside of Case) 


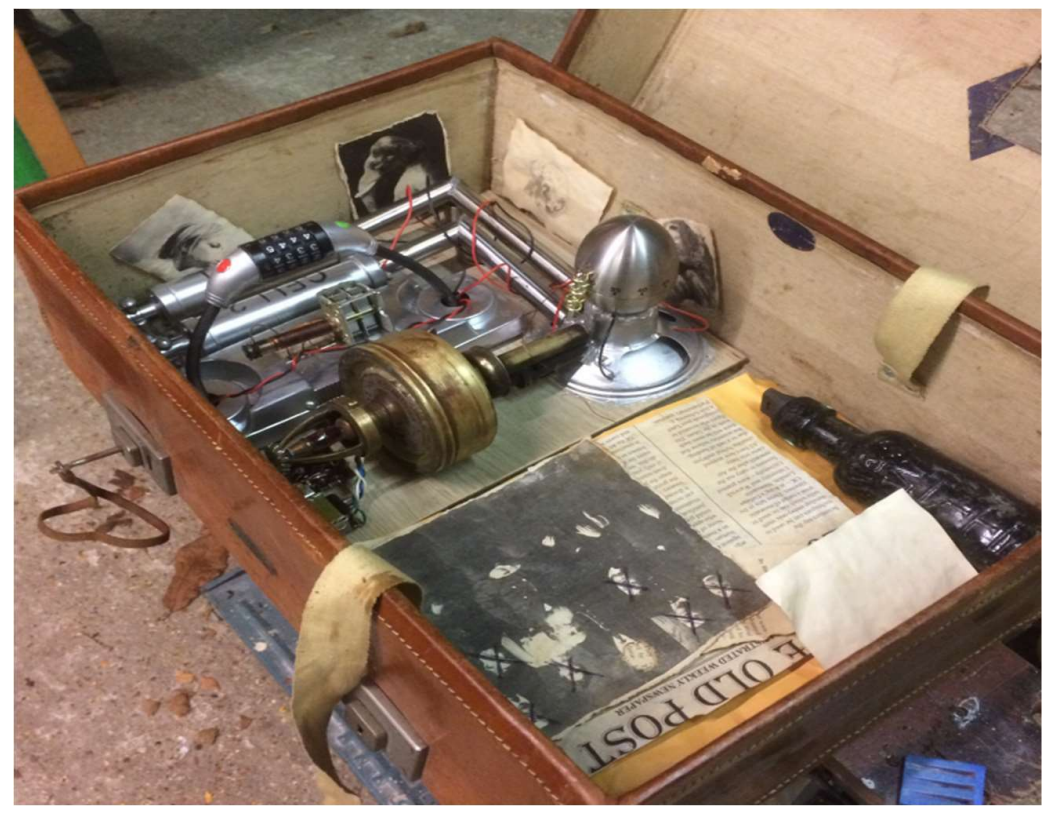

Figure 6 The Island of Dr. Moreau Prototype Game (Inside of Case)

Reflecting on the experience of using the Disruptive Media Learning Lab's escapED framework to develop the prototype, my development journey did deviate slightly from the framework's suggested course of action. In the framework, it is suggested that narrative/story should be defined quite early in the design and development process. However, personally I found that through my own development path, narrative/story design was left until the other steps of the framework had been completed. Although narrative design is considered an integral part of escape room design and playful learning, in this case, story was not considered to have a particularly big impact on either the learning aims or objectives behind the prototype experience. Once the other areas of the framework had been solidified, then inspiration for the experiences narrative came from the available equipment to build the activity with. In the case of the prototype game, a traditional style suitcase was considered perfect to create a self-contained activity. Additionally, the style of the case also gave inspiration for the time period that the narrative would be set, late 1800s to early 1900s, characters and basic plot, perhaps a traveler or doctor which could be used for the traditional "mad doctor" character. The choice for using "The Island of Dr. Moreau" as a theme was chosen predominantly because of a recent reading of the novel. The narrative enabled the use and adaptation of many materials and props that had already been obtained, mainly old junk, in order to embellish certain aspects of the puzzle activities. As an example, the third activity in the prototype game titled "The Test Subject", asked participants to locate a journal entry from a bibliographical citation. The puzzle had already been chosen but the narrative gave this a controlled situation to be done in. If a futuristic theme has been chosen for example, for the escape case then the activity might have been done slightly differently, perhaps participants would be asked to access the internet or search through ePub documents via a tablet device. This means for this games development, even though the narrative design came slightly later in the design process, it still played an active role in the development of the puzzles after they had been chosen.

The account presented on the use of the escapED framework for creating a prototype game to teach research methods, provides a first look at how the framework can and has been adapted to fit the particular needs of the developers in this case. The game is currently being tested with student and staff participants at Southampton University to assess whether it achieves its intended learning objectives and whether there are any engagement/motivation factors that it produces within the players.

It is observed that whilst most of the steps of the framework were followed in order, the developers deviated slightly from the process by choosing to design their games narrative as a final step. Whilst the authors believe that narrative design should come earlier in the development process as indicated in the framework, this is an interesting find in determining how others respond and utilise the escapED framework for their own purposes. Other studies are required to observe whether 
other developers follow the framework as intended or if there are similar observations where the steps are followed out of sync.

\section{Conclusions}

Presented in this paper has been an initial look at the feasibility and acceptance of embedding interactive GBL, particular to the case of educational escape rooms into higher education settings. Our findings so far have indicated that there is a high level of interest from higher education staff on how to develop these experiences for their own teaching practice. Whilst the authors acknowledge that the pilot sample size was small, the findings indicate that there is indeed an interest in this method, however, there is a need for a bigger study to validate support in higher education for the use of these examples of interactive GBL for learning. Other outcomes from the pilot suggest that more research and development needs to be done for accommodating larger cohorts of students as this was one of the main concerns raised by the pilot participants. Large numbers of students $(50+)$ were indicated by the participants as typical class sizes, so it was vital to ensure that all members of their class could be accommodated in a game experience if it was to be worked into their practice. Future works will include developing more game experiences with larger samples of higher education staff members for more detailed data of experience, player engagement and perceived educational value. Additionally, the pilot was conducted with participants who were all members of staff at Coventry University. The authors intend to conduct similar trials using external higher/further education facilitators to observe any similarities or differences towards reactions and engagement towards the method of educational escape rooms.

Following on from the pilot study, the paper has presented the theoretically informed escapED framework. The framework intends to provide educationalists and other interested parties a guideline to help develop and deliver educational escape rooms and other interactive GBL experiences of similar designs. Informed by Arnab \& Clarke's Trans-Disciplinary Methodology for Serious Games Design [29], and Nicholson's white paper research on entertainment escape rooms [27], the escapED framework is presented as a foundation in which key elements of GBL, pedagogic theory and entertainment games design and development processes are brought together into one easy to follow guideline. Further work will look to validate and provide assessment of how the framework is applied and whether it is considered a useful tool for aiding development of educational escape rooms for higher/further education.

Finally, the paper provides an account from a Professional Specialist in Learning Design based at an external University to which the escapED framework was developed. The account provides an overview of how the developer implemented the escapED framework to create an educational escape game for teaching research methods. Following the development journey of the developer in line with how they utilised the escapED framework, it has shown that more case studies are required to understand how the escapED framework is used as a design and development tool and whether the process suggested, needs to be modified to reflect actual development paths taken.

In this paper, the authors have presented the case that interactive GBL can be used to demonstrate to higher/further education staff how it is possible to develop and present learning challenges to students by considering game-play mechanics, dynamics and aesthetics so that students interact and produce understandable, fun and engaging results. Furthermore, it has been presented that a GBL product/resource can be easily developed and implemented, through engaging in an explicit process of brainstorming, finding out the needs of the audience, design, development, testing, sharing, and more as instructed through the escapED framework. Interactive GBL is presented as an approach that can help students and teachers break out of the lecture/test model and showcase what young learners can do, rather than create didactic testing platforms that try to catch them at what they can't do. The application of interactive GBL allows for a safe place for students to use different problem-solving styles, to add their own flair to the learning process, and to think about the impact that they could have on the world.

The escapED programme provides a holistic approach to developing learning practice and demonstrates a transition from a technology driven focus to a highly empathetic and experience and person centered approach, which has also emphasised on the potential of arts and creativity in enhancing the teaching and learning. The paper showcases the escapED framework as an approach that will inform the design of more contextually immersive learning experience, which can be adopted and adapted in various learning contexts. The approach taps into our natural tendencies to learn by playing. 


\section{References}

[1] Prensky, M., "'Fun, play and games: What makes games engaging," Digital game-based learning, vol. 5, pp. 1-05, 2001.

[2] Piccione, P.A., "'In search of the meaning of Senet," Archaeological Institute of America, 1980.

[3]. Parlett, D.S, "'The Oxford history of board games,", 1999.

[4] Huizinga, J., "'Homo ludens: proeve fleener bepaling van het spel-element der cultuur," Haarlem: Tjeenk Willink, 1948.

[5] Bekoff, M. and Allen, C., "'Intentional communication and social play: how and why animals negotiate and agree to play," Animal play: Evolutionary, comparative, and ecological perspectives, pp. 97-114, Cambridge, Cambridge University Press, 1998.

[6] Bateson, G., "A theory of play and fantasy." Psychiatr.Res.Rep., 2, pp. 39-51.

[7] Parikka, J., "'A geology of media,", 2015. Minneapolis, University of Minnesota Press. https://doi.org/10.5749/minnesota/9780816695515.001.0001

[8] Blascovich, J., and Bailenson, J. ,"'Infinite reality: Avatars, eternal life, new worlds, and the dawn of the virtual revolution,", William Morrow \& Co, 2011.

[9] Vorderer P., and Bryant, J., "'Playing video games: Motives, responses, and consequences,", London, Routledge, 2012.

[10] Newzoo Consultancy, "'Number of Video Gamers Worldwide in 2014, by Region (in Millions),", 2016 Report no. 04/13.

[11] Nichols, R. , "'The video game business,". Basingstoke, Palgrave McMillian, 2014.

[12] Tobias, S., Fletcher, J.D., and Wind, A.P., "'Game-based learning,", pp. 485-503.

[13] Steinkuehler, C., Squire, K,.and Sawyer, K., "'Videogames and learning," Cambridge handbook of the learning sciences, pp. 377-396. Sawyer, R.K. (ed.), Cambridge, Cambridge University Press, 2005.

[14] Whitton, N., "'Digital games and learning: Research and theory,", London, Routledge, 2014.

[15] Gee, J.P., "'Are video games good for learning," Nordic Journal of Digital Literacy, vol. 3, no. 10.

[16] Kafai, Y.B. and Resnick, M.,"Constructionism in practice: Designing, thinking, and learning in a digital world,", London, Routledge, 1996.

[17] Salen, K., "Gaming literacies: A game design study in action," Journal of Educational Multimedia and Hypermedia, vol. 16, no. 3, pp. 301., 2007.

[18] Prensky M., and Prensky, M. "'Digital game-based learning,", vol. 1, 2007.

[19] Squire, K., "Video Games and Learning: Teaching and Participatory Culture in the Digital Age. Technology, Education--Connections (the TEC Series).", 2011.

[20] Tang, S., Hanneghan, M., and El-Rhalibi, A."Pedagogy elements, components and structures for serious games authoring environment,", pp. 26-34. 5th International Game Design and Technology Workshop (GDTW 2007), Liverpool, UK. 2007.

[21] Tang, S., Hanneghan, M., and El-Rhalibi, A., "Introduction to games-based learning," Games Based Learning Advancements for Multi-Sensory Human Computer Interfaces.New York: IGI Global. 2009. https://doi.org/10.4018/978-1-60566-360-9.ch001

[22] Baker, A., Navarro, E.O. and Van Der Hoek, A., "An experimental card game for teaching software engineering processes," J.Syst.Software, vol. 75, no. 1, pp. 3-16, 2005. https://doi.org/10.1016/j.jss.2004.02.033

[23] Cook, E.D., and Hazelwood, A.C., "An active learning strategy for the classroom- "who wants to win... some mini chips ahoy?"," Journal of Accounting Education, vol. 20, no. 4, pp. 297306. 2002. https://doi.org/10.1016/S0748-5751(02)00012-X

[24] Whitehead, N., "Teaching use case modelling using Fluxx ${ }^{\circledR}, "$. Higher Education Academy, 2012.

[25] Linderoth, J., "Beyond the digital divide: An ecological approach to gameplay," Transactions of the Digital Games Research Association, vol. 1, no. 1. 2013.

[26] Kelley, T. and Kelley, D.,"Creative confidence: Unleashing the creative potential within us all," Glasgow, William Collins, 2013.

[27] Nicholson, S., "Peeking behind the locked door: A survey of escape room facilities.". White Paper available at http://scottnicholson. com/pubs/erfacwhite, 2015.

[28] Gygax, G., Arneson, D. and Hobbies, T., "Dungeons and dragons,", vol. 19, Lake Geneva, TSR, 1974.

[29] Arnab, S. and Clarke, S., "Towards a trans-disciplinary methodology for a game-based intervention development process," British Journal of Educational Technology. 2015 
pag. 86

[30] Sheldon, K.M. and Hilpert, J.C., "The balanced measure of psychological needs (BMPN) scale: An alternative domain general measure of need satisfaction," Motiv.Emotion, vol. 36, no. 4, pp. 439-451. 2012. https://doi.org/10.1007/s11031-012-9279-4 